\title{
A Simple 10-GHz Picosecond Pulse Source Based on Fiber Optical Parametric Oscillator
}

\author{
Yue Zhou, Kim K. Y. Cheung, Sigang Yang, P. C. Chui and Kenneth K. Y. Wong \\ Photonic Systems Research Laboratory, Department of Electrical and Electronic Engineering, \\ The University of Hong Kong, Pokfulam Road, Hong Kong \\ Phone: +(852) 28578483, Fax: +(852) 25598738, Email: kywong@eee.hku.hk
}

\begin{abstract}
We demonstrate a simple fully fiber-integrated picosecond optical parametric oscillator based on highnonlinear dispersion-shifted fiber. High quality picosecond pulses are generated with a 40-nm tuning range.
\end{abstract}

\section{Introduction}

Picosecond optical pulse sources have been playing an important role in ultrafast processes and optical communications. Picosecond pulse generators based on erbium-doped fiber lasers [1] and semiconductor lasers [2] have been investigated comprehensively. On the other hand, fiber optical parametic amplifier (FOPA) offers high gain and wide-gain bandwidth [3], which allows picosecond pulse generation in potential regions where practical lasers currently are not available.

Previous effort included utilizing parametric gain to generate picosecond pulse based on FOPA [4-5]. However, the availability of continuous wave (CW) lasers at certain wavelength limits the potentially tunability of such kind of picosecond source. Therefore it is highly desirable to explore its corresponding fiber optical parametric oscillator (FOPO) configuration, which does not require any seeding light.

Most of the picosecond FOPOs reported were pumped by picosecond lasers [6]. Since a picosecond laser is relatively expensive, it is desirable to build a picosecond generator just using a $\mathrm{CW}$ pump.

In this paper, we demonstrate a simple and economic picosecond optical parametric oscillator based on highlynonlinear dispersion-shifted fiber (HNL-DSF). We use a similar setup as previously reported actively modelocked FOPO [7]. However, the output pulsewidth of the current FOPO is measured to be 18 ps, narrower than that reported in [7]. The ring cavity is pumped with a sinusoidally modulated $\mathrm{CW}$ laser. The tuning range is from $1528 \mathrm{~nm}$ to $1548 \mathrm{~nm}$ and from $1564 \mathrm{~nm}$ to 1584 $\mathrm{nm}$, which is over $40 \mathrm{~nm}$. This scheme can be potentially useful in generating short pulse for ultra-fast communication.

\section{Experimental Setup}

The experimental setup of the FOPO is shown in Fig. 1. The pump was generated by a CW tunable laser source (TLS) with a fixed wavelength of $1556 \mathrm{~nm}$. The output of the laser was phase modulated by a phase modulator (PM) using a $10 \mathrm{GHz}$ pseudorandom binary sequence
(PRBS) with pattern length $2^{7}-1$ in order to increase the threshold for stimulated Brillouin scattering (SBS). The pump was then intensity modulated by a Mach-Zehnder modulater (MZM) driven by a $10 \mathrm{GHz}$ clock signal to produce a sinusoidally modulated pump. The polarization controllers (PCs), PC1 and PC2, were used to control the state of polarization (SOP) of the pump. After that, the pump was amplified by erbium-doped fiber amplifiers (EDFAs), EDFA1 and EDFA2, and filtered by a tunable bandpass filter (TBPF1) with a 1$\mathrm{nm}$ bandwidth to produce a high-power, low-noise pump. The average power of the pump was measured to be $0.5 \mathrm{~W}$ after the EDFA2. The pump then passed through a circulator (CIR), and the reflected power by SBS was monitored using a power meter. It was then coupled into the cavity for parametric amplification through the wavelength-division multiplexing coupler (WDMC1), which had passband wavelength from 1550 $\mathrm{nm}$ to $1559 \mathrm{~nm}$. Note that the cavity included a 400-m long HNL-DSF as the gain medium which had nonlinear coefficient of $14 \mathrm{~W}^{-1} \mathrm{~km}^{-1}$, zero-dispersion wavelength of $1554.7 \mathrm{~nm}$ and dispersion slope of $0.035 \mathrm{ps} / \mathrm{nm}^{2} / \mathrm{km}$.

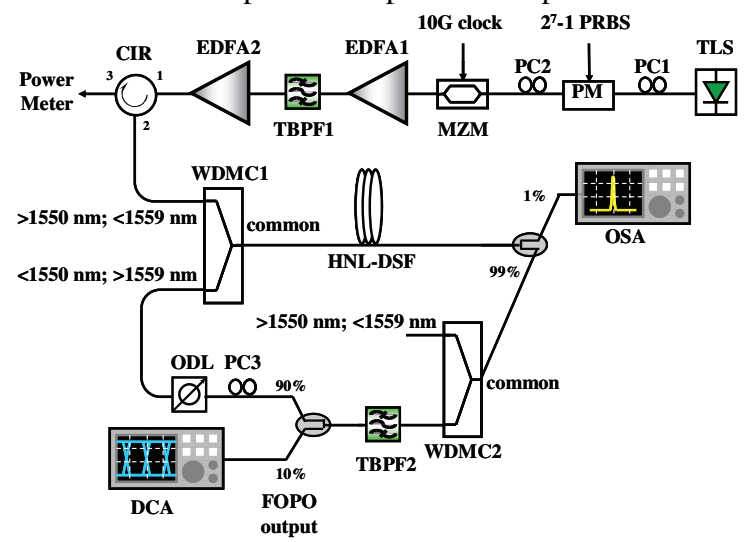

Fig. 1. Experimental setup of the FOPO.

The FOPO output spectrum was monitored by an optical spectrum analyzer (OSA) through a 1/99 coupler. WDMC2 was used to filter out the signal and idler by blocking the undesired pump. It was then filtered by TBPF2 with a bandwidth of $1 \mathrm{~nm}$. Tuning was achieved by adjusting the center wavelength of TBPF2. A 10/90 coupler in the cavity provided $90 \%$ feedback and $10 \%$ output. The feedback branch passed through the PC3 in the cavity, which was used to align the SOP of the signal (idler) with that of the pump, while the optical delay line 
(ODL) in the cavity was used to adjust the cavity length thus the pulsed signal can be synchronized with the pump. The output waveform of the signal (idler) was recorded using a digital communication analyzer (DCA).

\section{Results and Discussion}

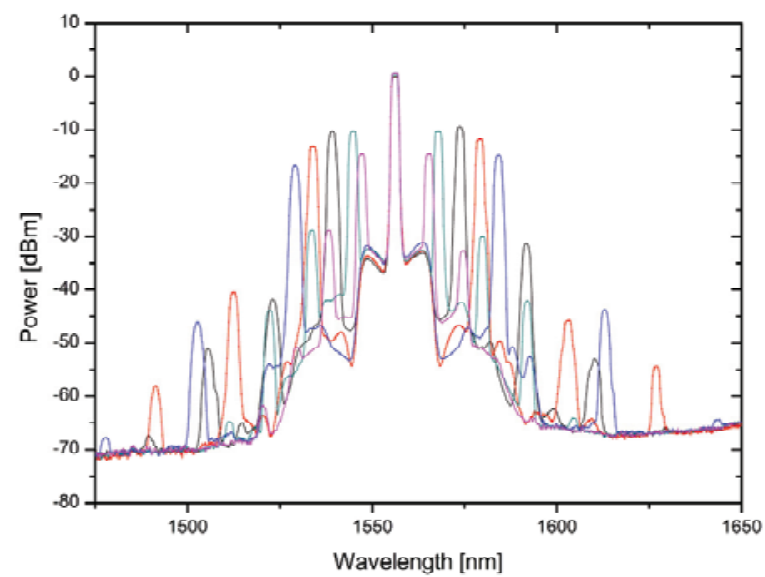

Fig. 2. Optical spectra measured at HNL-DSF output using OSA.

Fig. 2 shows the optical spectra measured at the HNLDSF output. The pump wavelength is fixed at $1556 \mathrm{~nm}$ the pump power is slightly adjusted to maintain narrow linewidth for all spectra. Wavelength tuning can be achieved by tuning the center wavelength of the TBPF2 inside the cavity. The achievable output tuning range is from $1528.5 \mathrm{~nm}$ to $1548.5 \mathrm{~nm}$ and from $1564.8 \mathrm{~nm}$ to $1584.9 \mathrm{~nm}$, which is over $40 \mathrm{~nm}$. The smaller peaks occurred are higher order parametric components. Further tuning is limited by the gain region of the FOPA when the pump wavelength is set at $1556 \mathrm{~nm}$. By changing the pump wavelength, we expect the tuning range can be much larger than this value, since the parametric gain allows signal (idler) to be generated at large frequency detune from the pump [3]. This kind of FOPO has a potentially wide tunability, since it does not require any seeding light, of which the availability at certain wavelengths limits the tuning range of FOPAbased pulse generator as reported in [4].

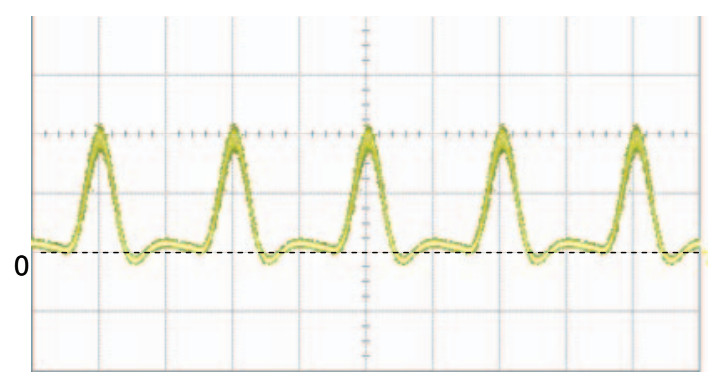

Fig. 3. Signal waveform observed at the FOPO output. Horizontal scale is $50 \mathrm{ps} /$ div.
Fig. 3 shows the waveforms of the output signal pulse at $1540 \mathrm{~nm}$ measured from the DCA. The repetition rate of the signal is measured to be $10 \mathrm{GHz}$, the same as the frequency of the sinusoidal clock source. The pulsewidth measured from the DCA is 18 ps and the jitter RMS is $870 \mathrm{fs}$, which is inherited from the clock source. The pulsewidth is narrower than that reported in [7].

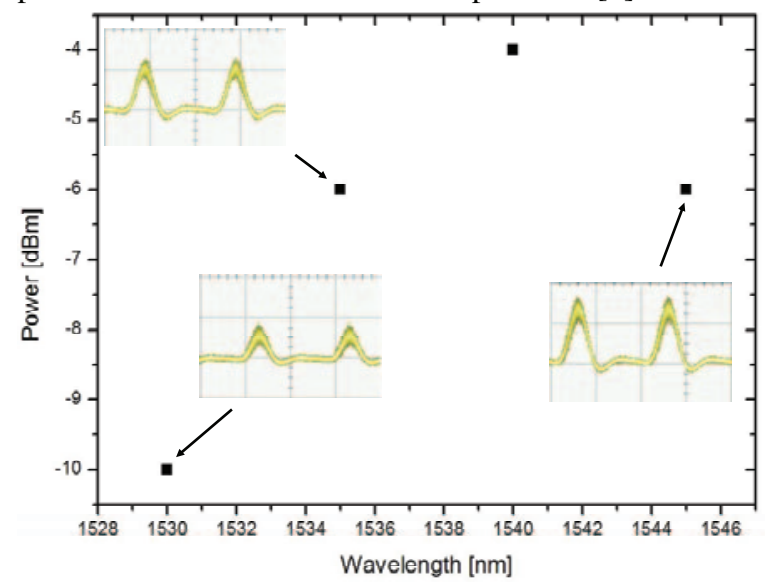

Fig.4. Output power of FOPO versus wavelength.

Fig. 4 shows the average power and waveform of the output pulse at four different wavelengths of $1530 \mathrm{~nm}$, $1535 \mathrm{~nm}, 1540 \mathrm{~nm}$ and $1545 \mathrm{~nm}$. The FOPO has largest output power at $1540 \mathrm{~nm}$, corresponding to the FOPA gain peak, while the output power decreases when the wavelength is tuned away from $1540 \mathrm{~nm}$ due to the gain spectrum of the FOPA.

\section{Conclusion}

In conclusion, an all-fiber picosecond optical parametric oscillator based on HNL-DSF was demonstrated. The output tuning range was over $40 \mathrm{~nm}$ and high quality pulse was generated with pulsewidth of 18 ps. This scheme could be found potentially useful in generating pulse for ultrafast communication.

\section{Acknowledgment}

The work described in this paper was partially supported by grants from the Research Grants Council of the Hong Kong Special Administrative Region, China (Project No. HKU 7172/07E and HKU 7179/08E). The authors would also like to acknowledge Sumitomo Electric Industries for providing the HNL-DSF.

\section{References}

1. A. Takada et al, Electron. Lett. 26 (1990) p.216

2. K. Otsuka et al, Opt. Lett. 16 (1991) p. 1759

3. M. E. Marhic et al, IEEE J. Sel. Top. Quantum Electron. 10 (2004) p.1133

4. T. Torounidis et al, J. Lightwave Technol. 23 (2005) p.4067 5. B. P. P. Kuo et al, in 2009 IEEE/LEOS Winter Topical Meetings, paper TuC3.5.

6. J. E. Sharping, J. Lightwave Technol. 26 (2008) p.2184

7. S. Yang et al, IEEE J. Sel. Top. Quantum Electron., accepted for publication. 